\title{
Interface-mediated extremely low thermal conductivity of graphene aerogel
}

\author{
Yangsu Xie ${ }^{\mathrm{a}}$, Shen $\mathrm{Xu}^{\mathrm{a}}$, Zaoli Xu ${ }^{\mathrm{a}}$, Hongchao Wu ${ }^{\mathrm{a}}$, Cheng Deng ${ }^{\mathrm{a}}$, Xinwei Wang ${ }^{\mathrm{a}}$, , * \\ a 2010 Black Engineering Building, Department of Mechanical Engineering \\ Iowa State University, Ames, IA 50011 \\ ${ }^{\mathrm{b}}$ School of Urban Development and Environmental Engineering, \\ Shanghai Second Polytechnic University, Shanghai 201209, P.R. China
}

\begin{abstract}
Due to the ultra-high thermal conductivity $(\kappa)$ of graphene, graphene-based materials are expected to be good thermal conductors. Here, however, we uncovered extremely low $\kappa$ of ultralight graphene aerogels (GAs). Although our GA $\left(\sim 4 \mathrm{mg} \cdot \mathrm{cm}^{-3}\right)$ is about two times heavier than air $\left(\sim 1.2 \mathrm{mg} \cdot \mathrm{cm}^{-3}\right)$, the $\kappa\left(4.7 \times 10^{-3}-5.9 \times 10^{-3} \mathrm{~W} \cdot \mathrm{m}^{-1} \cdot \mathrm{K}^{-1}\right)$ at room temperature (RT) is about $80 \%$ lower than that of air $\left(0.0257 \mathrm{~W} \cdot \mathrm{m}^{-1} \cdot \mathrm{K}^{-1}\right.$ at $\left.20^{\circ} \mathrm{C}\right)$. At low temperatures, the GA's $\kappa$ reaches a lower level of $2 \times 10^{-4}-4 \times 10^{-4} \mathrm{~W} \cdot \mathrm{m}^{-1} \cdot \mathrm{K}^{-1}$. This is the lowest $\kappa$ ever measured to our best knowledge. The mechanism of this extremely low $\kappa$ is explored by studying the temperature variation of GA's $\kappa$, thermal diffusivity $(\alpha)$ and specific heat $\left(c_{p}\right)$ from RT to as low as $10.4 \mathrm{~K}$. The uncovered small, yet positive $\partial \alpha / \partial T$ reveals the dominant interface thermal contact resistance in thermal transport. For normal materials with thermal transport sustained by phononphonon scattering, $\partial \alpha / \partial T$ always remains negative. The study of $c_{p}$ suggests highly disordered and amorphous structure of GAs, which also contributes to the ultralow $\kappa$. This makes the GA a very promising thermal insulation material, especially under vacuum conditions (e.g. astronautics areas).
\end{abstract}

\footnotetext{
${ }^{*}$ Corresponding author. Tel: +1 515 2948023. E-mail address: xwang3@ iastate.edu
} 


\section{Introduction}

To facilitate graphene's wide applications, three-dimensional graphene-based aerogels (GAs) with self-assembled microstructures has become one of the most distinctive and promising forms. Compared to two-dimensional graphene sheets or one-dimensional carbon nanotubes, 3-D graphene aerogel is superior with flexible shape, strong mechanical strength, lightweight, high porosity and excellent durability. These advantages fulfill the requirements of industrial applications and are expected to pave the way for novel applications of graphene. Extensive work has been done and remarkable progress has been made for its applications in electrochemical devices, ${ }^{1,2}$ environmental treatment ${ }^{3,4}$ and energy storage, ${ }^{5}$ etc. Li et al. demonstrated that their GA synthesized by chemical reduction with ethylenediamine (EDA) is a highly efficient and recyclable absorbent for organic liquids; ${ }^{6} \mathrm{Xu}$ et al. measured the reversible magnetic field-induced strain and strain-dependent electrical resistance of GA decorated with $\mathrm{Fe}_{3} \mathrm{O}_{4}$ nanoparticles, proving it has potential applications as an ultralight magnetic elastomer. ${ }^{7}$ Zhang et al. presents the fabrication and characterization of three-dimensional (3D) GApolydimethylsiloxane (PDMS) composites. Their outstanding electrical, thermal and mechanical properties propose potential applications in stretchable electronic devices, ultralarge strain sensors, thermal interface materials, hydrophobic anti-icing films, and energy absorption and viscoelastic damping devices. ${ }^{8}$

As a synthetic highly porous material, aerogel is derived from a gel by replacing the liquid inside it with air. Due to the high porosity and randomly oriented microstructure, aerogels have always been highly insulating materials with a thermal conductivity lower than still air. ${ }^{9}$ Before GA, the most typical aerogel is silica aerogel, which is known as the best insulator so far. Since 2004, 
large amount of aerogels are produced and utilized as building insulation materials. ${ }^{10}$ The thermal conductivity of silica aerogel has been reported as low as $0.02-0.036 \mathrm{~W} \cdot \mathrm{m}^{-1} \cdot \mathrm{K}^{-1}$ in atmospheric pressure ${ }^{11,12}$ to $0.004 \mathrm{~W} \cdot \mathrm{m}^{-1} \cdot \mathrm{K}^{-1}$ in moderate vacuum $(0.003 \mathrm{~atm}) .{ }^{13}$ For other kinds of inorganic aerogels, such as metal oxide aerogels, the thermal insulating ability is comparatively poor. For example, the thermal conductivity of alumina aerogel was measured to be 29,98 and $298 \mathrm{~m} \mathrm{~W} \cdot \mathrm{m}^{-1} \cdot \mathrm{K}^{-1}$ at temperatures of $30{ }^{\circ} \mathrm{C}, 400{ }^{\circ} \mathrm{C}$ and $800{ }^{\circ} \mathrm{C}$ respectively. ${ }^{14}$ Compared to its peers, GAs is expected to be a more outstanding thermal insulating material considering its high porosity, flexible and strong mechanical properties and the controllable functional groups at the surfaces. Fan et al. investigated the impact of thermal annealing on the thermal conductivity of GAs. Their GAs with a density of 14.1-52.4 $\mathrm{mg} \cdot \mathrm{cm}^{-3}$ was synthesized by a chemical reduction method and dried with supercritical $\mathrm{CO}_{2}$. The value for the measured thermal conductivity at RT was $0.12-0.36 \mathrm{~W} \cdot \mathrm{m}^{-1} \cdot \mathrm{K}^{-1},{ }^{15}$ which is remarkably low compared with the ultrahigh thermal conductivity of graphene. ${ }^{16}$ Another research group studied the effect of different reducing agents and thermal annealing on the properties of GAs. Their GAs with a density of $16-41 \mathrm{~kg} \cdot \mathrm{m}^{-3}$ have a thermal conductivity of about $0.1 \mathrm{~W} \cdot \mathrm{m}^{-1} \cdot \mathrm{K}^{-1} \cdot{ }^{17}$ However, these numbers still show little advantage over other aerogels reviewed above in terms of insulation. Wicklein et al. synthesized a strong anisotropic foam by freeze-casting suspensions of cellulose nanofibers, graphene oxide and sepiolite nanorods. The material is lightweight $\left(7.5 \mathrm{~kg} \cdot \mathrm{m}^{-3}\right)$, super-insulating (with a thermal conductivity of $15 \mathrm{~mW} \cdot \mathrm{m}^{-1} \cdot \mathrm{K}^{-1}$ ), and fire retardant, which is very promising as an advanced high-performance thermally insulating material. ${ }^{18}$

Among the various developed methods for synthesizing GAs, self-assembling by chemical reduction from GO aqueous suspensions attracts wide attentions. ${ }^{19,20}$ Compared to other method 
for synthesizing graphene aerogel, the chemical reduction process is comparatively simple and effective. Different functional groups are introduced onto the surface of the GO flakes during chemical reduction. By carefully controlling the various functional groups, different desired properties of the resulting GAs can be achieved and thus facilitate GAs' application in different areas. Sun $e t$ al. ${ }^{21}$ synthesized GA with a density as low as $0.16 \mathrm{mg} \cdot \mathrm{cm}^{-3}$, which crowns GA as the lightest material in the world. They employed direct lyophilization to remove the solvent first and then fed hydrazine vapor for chemical reduction, followed by vacuum drying. However, the synthesis process is dangerous and difficult to operate considering the use of hydrazine vapor, which is highly toxic. Another reduction agent-EDA was also reported for GA assembling. ${ }^{6,22}$ From their study, EDA is efficient in preventing volume shrinkage and improving the mechanical compressibility of the hydrogel. The resulting GA produced from chemical reduction and freeze-drying is electrically conductive and mechanically strong.

In this work, based on Hu et al.'s method, ${ }^{22}$ we present a modified synthesizing method of GAs. We report a record-low thermal conductivity $(\kappa)$ for our ultralight GAs $\left(\sim 4 \mathrm{mg} / \mathrm{cm}^{3}\right)$ under vacuum. The $\kappa$ of our GAs is down to $2 \times 10^{-4}-4 \times 10^{-4} \mathrm{~W} \cdot \mathrm{m}^{-1} \cdot \mathrm{K}^{-1}$ at low temperatures $(\sim 40 \mathrm{~K})$ and $4.7 \times 10^{-3}-5.9 \times 10^{-3} \mathrm{~W} \cdot \mathrm{m}^{-1} \cdot \mathrm{K}^{-1}$ at room temperature $(\mathrm{RT})$, which makes it a very promising material for extreme thermal insulation. The real thermal diffusivity and electrical resistivity of GAs are reported and discussed in detail to understand the underlying physical principles for the ultralow thermal conductivity.

\section{Sample synthesizing method and characterization}

The graphene oxide dispersion in water (purchased from Graphene Supermarket) has the 
concentration of $5 \mathrm{~g} / \mathrm{L}$ and $\mathrm{C} / \mathrm{O}$ ratio of about 4 . Ethylenediamine (EDA) anhydrous (99.9\%) was obtained from Fisher Scientific, and used as received. $4 \mathrm{uL}$ reducing agent ethylenediamine (EDA) is diluted with deionized (DI) water $(2 \mathrm{~mL})$ under magnetic stirring. In this work, the GO solution $(5 \mathrm{mg} / \mathrm{mL} \times 3 \mathrm{~mL})$ is added into the EDA solution drop by drop during the magnetic stirring. After 30 minutes of medium-high speed magnetic stirring, the GO solution is partly reduced and the GO-EDA mixture becomes uniform. Then the mixture is sealed and heated in an oven at $95{ }^{\circ} \mathrm{C}$ for 6 hours. The dispersion of $\mathrm{GO}$ first becomes brown colloidal and finally transforms into a black hydrogel. During this process, the GO flakes assemble into a macroscopic hydrogel with little stacking. EDA as a reduction agent leads to ring-opening reaction of epoxy groups and functionalization on the surface of graphene oxide. Meanwhile, the graphene oxide is partly reduced by restoring part of the $s p^{2}$ regions. ${ }^{22}$ The resulting hydrogel exhibits no volume shrinkage during the heating. After that, the hydrogel is subjected to freezedrying for 48 hours, so as to completely remove the solvent inside the sample. During the freezing process, cells are made with the formation of ice crystals pushing the r-GO sheets together into cell walls. After freeze-drying, a black aerogel sample is formed. Then the sample is put at the bottom of a long quartz tube, and then flushed with Argon gas for 2 hours to remove air inside the sample completely. After that, the tube is sealed and a microwave heating process (1-5 minutes) is employed. The microwave heating removes a large number of residual functional groups. The conjugation of $s p^{2}$ and the $\pi-\pi$ interaction are restored. ${ }^{22}$

The final graphene aerogel sample has metal grey color and good mechanical strength. The GO/EDA ratio and microwave heating time can be varied to make GAs samples with different density and mechanical properties. The volume of the hydrogel is mainly determined by the 
GO/EDA ratio. Too small or too large ratio will lead to a volume shrinkage after the heating. If the EDA amount is not sufficient to functionalize all of the graphene oxide flakes, the graphene oxide cannot assemble into an integrated hydrogel. If the EDA amount is too large, the $\mathrm{pH}$ value of the suspension will be too high to keep the GO colloids stable, which results in a shrinkage of the resulting hydrogel. ${ }^{22}$ If we increase the GO and EDA amount but keep a suitable $\mathrm{pH}$ value (around 11.5), the resulting GA will be much denser and the mechanical strength improves accordingly. We found that when the GO concentration is less than $1.7 \mathrm{mg} / \mathrm{mL}$, the sample presented some volume shrinkage. Thus the obtained minimum density of the GAs sample is about $2 \mathrm{mg} \cdot \mathrm{cm}^{-3}$. Improved mechanical strength of the resulting samples can be achieved by increasing the GO concentration and EDA amount.

The structure of the GAs is characterized by scanning electron microscope (SEM). The SEM images were taken by using an FEI Quanta 250 field emission SEM with a voltage of $8.00 \mathrm{kV}$. Under SEM, the self-assembled foam-like network can be seen clearly [Figure 1(a)-(b)]. The cell walls of GAs are made up of reduced GO (r-GO) sheets. The thin r-GO sheets fold, curve, twist, and interconnect with adjacent sheets, constituting the framework with pores of tens to hundreds of $\mu \mathrm{m}$. The Raman Spectra of GAs [Figure 1 (c)] exhibits two pronounced peaks at about 1348 and $1585 \mathrm{~cm}^{-1}$, corresponding to the $\mathrm{D}$ band and $\mathrm{G}$ band. The $\mathrm{G}$ band reflects the $s p^{2}$ carbon. Its intensity can be used to analyze the level of graphitization in GAs. The D band sources from the defects and disorder structure in the $s p^{2}$ domains. ${ }^{23}$ Our GAs show an $I_{D} / I_{G}$ ratio of 1.14 , which is higher than the $I_{D} / I_{G}=1.06$ for GO. Increased $I_{D} / I_{G}$ is commonly reported for GA synthesized from chemical reduction. ${ }^{23-25}$ Although the microwave heating removes most of the residual functional groups from GA, the chemical reduction by EDA brings in a large number of disorder 
in the $s p^{2}$ domains. As the residual functional groups are removed during chemical reduction, some in-plane $\mathrm{C}=\mathrm{C}$ bond cracks and a large number of defects are generated. This is the main reason for the increase of $I_{D} / I_{G}$. Besides, it has been reported that the increased $I_{D} / I_{G}$ is related to the average size of the $s p^{2}$ domains. ${ }^{26}$ During the microwave reduction, numerous new graphitic domains with smaller size might be created, which makes the averaged $s p^{2}$ domain size decrease. ${ }^{24}$ It is possible that the microwave heating reduction contributes to a small decrease of $I_{D} / I_{G}$, but the overall reduction process still shows an increase of $I_{D} / I_{G}$. This phenomenon has also been reported in Hu et al's work. ${ }^{22}$ The Raman spectrum of GA is obtained using Olympus BX51 universal research Microscopy under $4 \times$ lens, with $8 \mathrm{~mW}$ laser power, and 5 minutes integration. For comparison, the Raman spectrum of GO flakes $(100 \times$ lens, $3.0 \mathrm{~mW}$ laser power and 60s integration) and the Raman spectrum of GF $(100 \times$ lens, $0.8 \mathrm{~mW}$ laser power and $10 \mathrm{~s}$ integration) are also presented.

The x-ray diffraction (XRD) is conducted with a Siemens D500 x-ray diffractometer using Cu xray tube operated at $45 \mathrm{kV}$ and $30 \mathrm{~mA}$. XRD patterns of GAs [figure 1 (d)] shows three major peaks at about $17.590^{\circ}, 21.345^{\circ}$ and $26.376^{\circ}$, yielding an interlayer spacing of 3.395-5.065 based on the fitting, which is a little larger than the $3.36 \AA$ from graphite's (002) plane while much smaller than the $8.32 \AA$ from GO's $10.6^{\circ} 2 \theta$ peak. ${ }^{27}$ The decreased interlayer spacing from GO to GA demonstrates the removal of large amount of the oxygen-containing functional groups of GO. The larger interlayer spacing of GAs than that of graphite indicates the existence of residual functional groups at the surface of GAs, which makes the r-GO sheets inside GAs different from graphene. As the reduction going on, the peak of GA is expected to shift from the peak of graphene oxide at $10.27^{\circ}$ to that of graphite at $26.7^{\circ} .^{28,29}$ Therefore, the peak at $21.345^{\circ}$ is 
due to the presence of partly reduced graphene oxide. The peak at $21.345^{\circ}$ is sharp, indicating that there is a short-range order. The XRD result of graphene foam material is also plotted in figure 1 (d) for comparison. The peaks of GF are very sharp and have high intensity, which is a typical XRD pattern of well-crystalline graphene; while the peaks of GA are wide and the base line is irregular with large noises. This indicates the large percent of amorphous structure in the GA.

Chemical analysis of GAs is conducted by x-ray photoelectron spectroscopy (XPS) on a PHI55000 XPS with an Al Ka source (1486.6 eV). Specifically, the survey spectra [figure 1 (e)] were collected from 0 to $1100 \mathrm{eV}$ with a pass energy of $187.85 \mathrm{eV}$ and a step size of $0.8 \mathrm{eV}$; high-resolution spectra for specific elements were acquired with a pass energy of $58.70 \mathrm{eV}$ and a step size of $0.25 \mathrm{eV}$. The resulting elemental composition of the GAs is $\mathrm{C}(82.05 \%), \mathrm{N}(6.99 \%)$, $\mathrm{O}(9.68 \%), \mathrm{Na}(0.56 \%)$, and $\mathrm{Si}(0.71 \%)$. Figure 1 (f) shows the C1s XPS spectrum, which has four obvious peaks by deconvolution, corresponding to the $\mathrm{C}-\mathrm{C}, \mathrm{C}-\mathrm{O}$, and $\mathrm{C}=\mathrm{O}$ and $\mathrm{C}(\mathrm{O}) \mathrm{OH}$ bond respectively. The $\mathrm{C}-\mathrm{C}$ bond takes the majority, while other bonds also exist. Figure 1(g) presents the deconvoluted N1S spectrum of GAs. The fitting of the spectrum gives the following peaks: the highest peak $\mathrm{N} 1$ at $398.5 \mathrm{eV}$ represents the pyridinic nitrogen; $\mathrm{N} 2$ at $400.5 \mathrm{eV}$ is attributed to the pyrrolic-type nitrogen; $\mathrm{N} 3$ at $403.7 \mathrm{eV}$ corresponds to the oxidized nitrogen. ${ }^{6,30}$ The high porosity endows GAs with an ultralow density $\left(2-6 \mathrm{mg} \cdot \mathrm{cm}^{-3}\right.$ depending on the microwave heating time as well as the ratio of GO and EDA), which is comparable with that of air $\left(1.28 \mathrm{~kg} / \mathrm{m}^{3}\right)$. Figure $1(\mathrm{~h})$ shows one ultralight GA sample standing on a dandelion. The dandelion has very little deformation under the weight of the sample. The GAs have good mechanical strength and elasticity. Figure 1 (i) shows the compressibility test. After being 

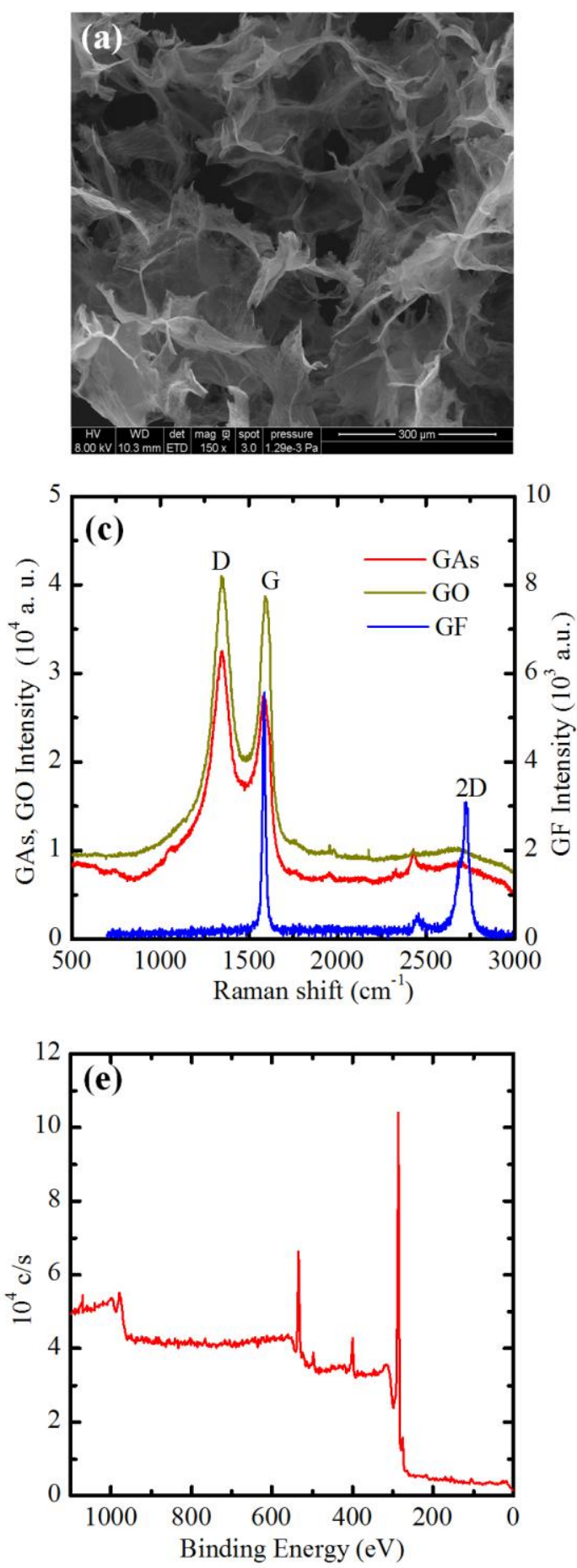
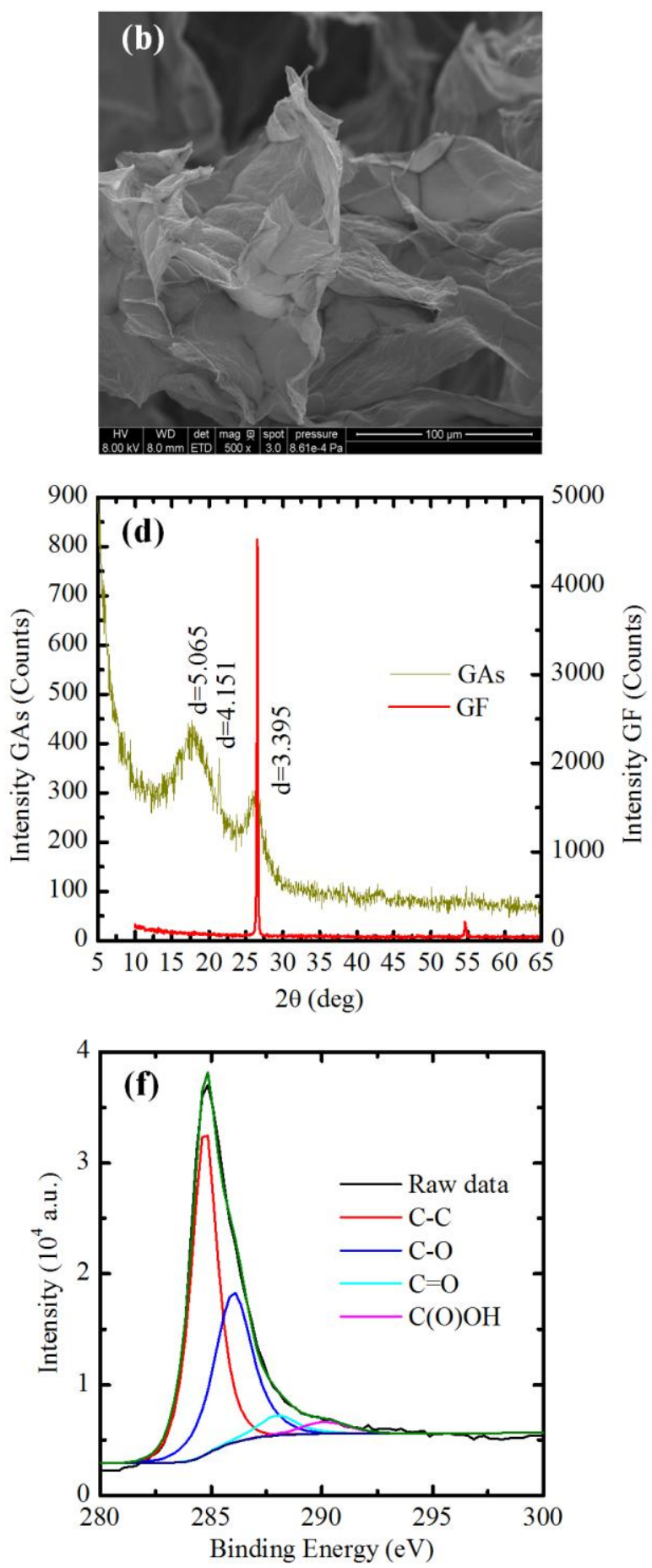

Figure 1. (to be continued in the next page) 

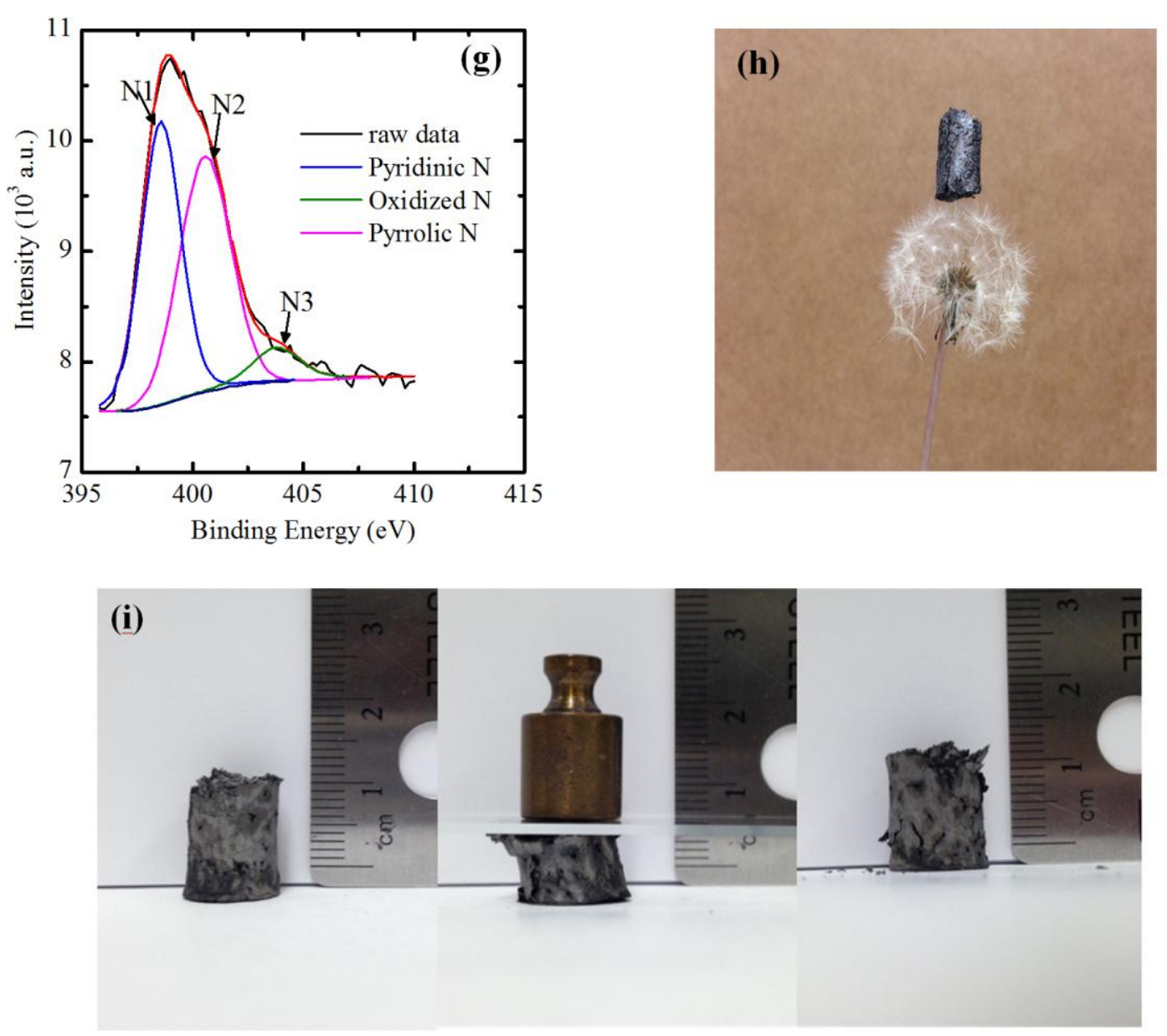

Figure 1. (a) and (b): SEM images of GAs cellular and wall structure. (c): Raman spectrum. (d): XRD spectrum. (e) X-ray photoelectron spectra of GA. (f) The XPS C1s spectrum, indicating different bonds for carbon atoms in the sample. (g) The XPS N1s spectrum, suggesting the presence of the pyridinic (N1), pyrolic and quaternary nitrogen (N2), and pyridine-N-oxide (N3) at the sample surface. (h) A GA on a dandelion. (i) The compressing test with a 10-gram weight. 
removed with a 10-gram weight, the GA sample (density $4 \mathrm{mg} / \mathrm{cm}^{3}$ ) completely recovers from the deformation. This shows the good elasticity and compressibility of our GA material.

\section{Methods for thermal characterization}

3.1 The transient electro-thermal technique

The thermal diffusivity of GAs samples at different temperatures are measured using the transient electro-thermal (TET) technique which is developed by our laboratory. The TET technique is an accurate and reliable approach to measuring thermal diffusivity of various solid materials. A Janis closed cycle refrigerator (CCR) system is utilized to provide stable environmental temperatures from $295 \mathrm{~K}$ to $10 \mathrm{~K}$. The GA sample is suspended between two gold-coated silicon electrodes on a thin glass wafer. Two smooth silicon wafers of smaller size are carefully placed on the sample edges and compressed tightly by clips and epoxy resin. In this way, the thermal contact resistance can be reduced to a negligible level. The sample is then placed on the stage of cold head. A small amount of silver paste is applied to connect the electrodes to the wirings. The whole stage is then shielded with a radiation shield and sealed with a clamped vacuum chamber. For data collecting, a step current is fed through the GAs sample by a current source. An oscilloscope is used to record the resulting voltage-time $(V-t)$ profile. Figure 2 (a) shows the schematic of the experiment set-up. The vacuum jacket is pumped to a pressure lower than 0.5 mTorr during the whole measurement to reduce the heat convection to a negligible level.

TET measurements are conducted every $25 \mathrm{~K}$ at environmental temperature from $295 \mathrm{~K}$ to 100

K. Denser data points (every 5-20 K) are collected at low temperatures $(<100 \mathrm{~K})$ to have a 
clearer view of low temperature effects. Details for the physical model of TET can be found in our previous paper. ${ }^{31}$ For the suspended samples, the one dimensional heat transfer model in the length direction is reasonable. Heat convection and gas conduction are neglected considering the very low air pressure $(<0.5 \mathrm{mTorr})$ in the chamber. The average normalized temperature rise, which is defined as $T^{*}=\left[T(t)-T_{0}\right] /\left[T(t \rightarrow \infty)-T_{0}\right]$, is given as:

$$
T^{*}=\frac{48}{\pi^{4}} \sum_{m=1}^{\infty} \frac{1-(-1)^{m}}{m^{2}} \frac{1-\exp \left[-m^{2} \pi^{2} \alpha_{\text {measure }} t / L^{2}\right]}{m^{2}},
$$

where $\alpha_{\text {measure }}=(1-f) \alpha$ is the measured thermal diffusivity. $\alpha$ is the real thermal diffusivity of GA. $f$ is a type of Biot number which represents the heat loss from the surfaces of the sample by radiation. The temperature evolution of the sample results in the voltage change of the sample: $T^{*}$ $=\left(V_{\text {sample }}-V_{0}\right) /\left(V_{\infty}-V_{0}\right)$, with $V_{0}$ and $V_{\infty}$ are the voltage of the sample before and after the heating respectively. Hence, by measuring the voltage evolution, we can obtain the normalized temperature profile. Here $f$ can be defined as $-8 \varepsilon_{r} \sigma T_{0}{ }^{3} L^{2} / D \pi^{2} \kappa$. Thus, the measured thermal diffusivity becomes:

$$
\alpha_{\text {measure }}=\alpha+\frac{1}{\rho c_{p}} \frac{8 \varepsilon_{r} \sigma \bar{T}^{3}}{D} \frac{L^{2}}{\pi^{2}}
$$

$\rho c_{p}$ is the volumetric specific heat; $\varepsilon_{r}$ is the emissivity; $\sigma$ is the Stephen-Boltzmann constant; $\bar{T}$ is the average temperature during the joule heating; $L$ and $D$ are the length and thickness of the sample respectively. From this equation, if other parameters are kept constant, the measured thermal diffusivity of a sample is linearly proportional to $L^{2}$. TET experiments are repeated to the same GA sample at two or three different lengths (Table 1). For each group, a rectangular sample is cut from an equal-thickness film of GA. For group 1, a sample is measured three times with different lengths, denoted as sample 1-1, 1-2 and 1-3 respectively; for group 2, another sample is measured twice, denoted as sample 2-1 and 2-2 respectively. 
Table 1. Details of GA samples characterized

\begin{tabular}{l|c|c|c|c|c}
\hline Sample index & $1-1$ & $1-2$ & $1-3$ & $2-1$ & $2-2$ \\
\hline Group & 1 & 1 & 1 & 2 & 2 \\
\hline Length $[\mathrm{mm}]$ & $4.90 \pm 0.01$ & $3.40 \pm 0.02$ & $2.60 \pm 0.05$ & $4.90 \pm 0.02$ & $2.80 \pm 0.01$ \\
\hline Width $[\mathrm{mm}]$ & $1.70 \pm 0.11$ & $1.70 \pm 0.09$ & $1.70 \pm 0.12$ & $2.02 \pm 0.07$ & $2.20 \pm 0.05$ \\
\hline Density $\left[\mathrm{mg} \cdot \mathrm{cm}^{-3}\right]$ & $4.20 \pm 0.38$ & $4.20 \pm 0.38$ & $4.20 \pm 0.38$ & $3.90 \pm 0.36$ & $3.90 \pm 0.36$ \\
\hline
\end{tabular}

The electrical resistivity of GA is not linearly dependent on temperature, as indicated in Figure 4 (b). But in our TET measurement, the temperature increase of the sample induced by joule heating is very small $(\Delta T<6 \mathrm{~K})$. In this very small temperature range, the linear relationship between resistance and temperature rise can be assumed justifiably. The decreasing resistance profile is linearly reflected in the decreasing voltage during the step current. The recorded experimental $V-t$ data is theoretically fitted by using different trial values of the thermal diffusivity. By using equation (1) and MATLAB programming, the experimental data is fitted by comparing with the theoretical curve with different trial value of measured thermal diffusivity $\left(\alpha_{\text {measure }}\right)$. Applying the least square fitting technique, the value giving the best fit of $V_{\exp }$ is taken as $\alpha_{\text {measure }} \alpha_{\text {measure }}$ represents the thermal diffusivity during the joule heating process. The corresponding real temperature $(T)$ should be the average temperature during the heating process. Figure 2 (b) shows the normalized temperature profiles for sample 1-1 at different environmental temperatures: $295 \mathrm{~K}, 95 \mathrm{~K}$, and $10 \mathrm{~K}$. The experiment data agrees very well with the theoretical value calculated from equation (1). As the temperature decreases from $295 \mathrm{~K}$ to $10 \mathrm{~K}$, the time to reach the steady state becomes longer and longer, which indicates that the thermal diffusivity is decreasing with the lowered temperature. The profile of the thermal diffusivity against 
temperature is discussed in the last section of this work. To determine the uncertainty of the fittings, different trail values are used for the fitting. It is found that when the trial values are changed by $\pm 10 \%$, the fitting curve deviates obviously from the experimental data. Thus the fitting uncertainty is estimated as $10 \%$, but the real error should be much smaller since we measure each value of thermal diffusivity for more than 30 times and take the average value as the final thermal diffusivity.

\subsection{The steady-state electro-thermal technique}

The thermal conductivity $\left(\kappa_{m}\right)$ of GAs is measured using the steady-state electro-thermal (SET) technique from RT to $10 \mathrm{~K}$. When temperature of the sample becomes stable, the governing equation for energy balance can be expressed as:

$$
\kappa \frac{\partial^{2} T(x)}{\partial x^{2}}+q_{0}=0
$$

in which $\kappa$ is the effective thermal conductivity which includes the radiation effect, $T(x)$ is the temperature at $x$ position, and $q_{0}=I^{2} R_{1} / A_{c} L$ is the joule heating rate per unit volume. $I$ is the current applied to the sample, $R_{1}$ is the resistance at the steady state, $A_{c}$ and $L$ are the crosssection area and the length of the sample respectively. Solving the governing equation, the temperature distribution is obtained as $T(x)=-q_{0}\left(x^{2}-L x\right) / 2 \kappa+T_{0}$. The average temperature along the sample is $\bar{T}(x)=\int_{x=0}^{L} T(x) d x / L=T_{0}+q_{0} L^{2} / 12 \kappa$. Thus, the average temperature rise is $\Delta T=$ $I^{2} R_{1} L / 12 \kappa A_{c}$. The temperature change reflects in the resistance change as $\Delta T=\Delta R /(d R / d T)$, in which $\Delta R$ is the resistance change before and after the heating. $d R / d T$ is obtained by differentiating the $R-T$ curve. Figure 2 (c) shows the $d R / d T$ profiles. Combing the two equations, we obtain the effective thermal conductivity as: 

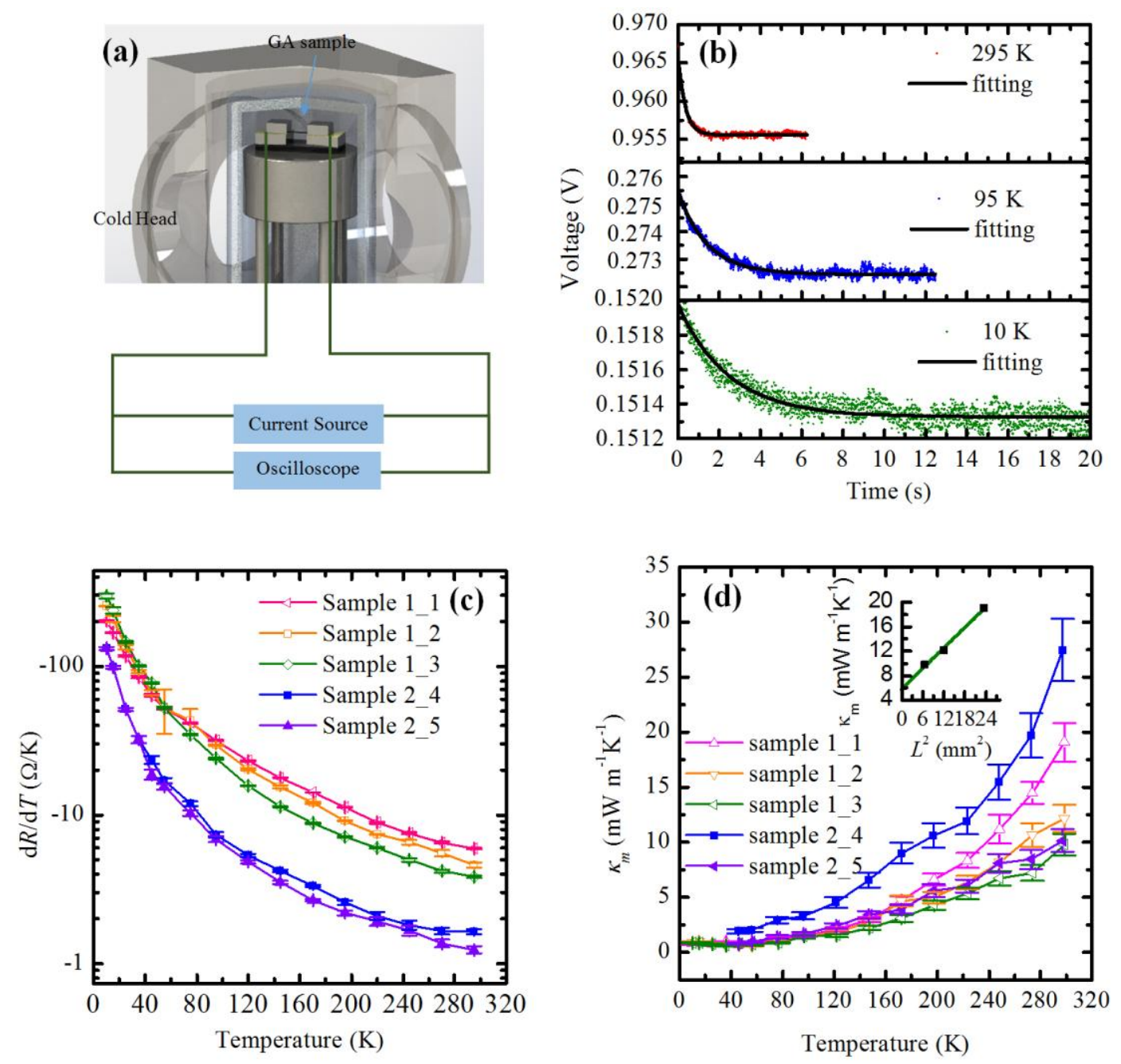

Figure 2. (a) The schematic of the experiment set up. (b) The normalized temperature profiles for sample 1-1 at different temperatures: $295 \mathrm{~K}, 95 \mathrm{~K}$ and $10 \mathrm{~K}$. The symbols are experimental data and the lines are the theoretical fittings. (c) The first derivative of electrical resistance against temperature profiles obtained by differentiating $R-T$ curve. Some errors are less than $\pm 2 \%$, so they are not very visible. (d) The measured thermal conductivity of the five GA samples in this work. The inset shows one of the examples for the linear fitting (for group 1 at real temperature of $298.6 \mathrm{~K}$ ) to obtain its real thermal conductivity. 


$$
\kappa_{m}=\frac{I^{2} R_{1} L}{12 A_{c} \cdot \Delta R} \cdot \frac{d R}{d T} .
$$

The $\kappa_{m}$ should represents the thermal conductivity at steady state, thus the real temperature corresponding to $\kappa_{m}$ is: $T_{1}=T_{0}+\Delta R /(d R / d T)$. Figure 2 (d) shows the measured thermal conductivity of the five samples (three in group 1 and two in group 2). The error is calculated by using the error transfer theory.

To subtract the radiation effect, each sample is measured 2-3 times to obtain the thermal diffusivity in different lengths. The sample details are summarized in Table 1. For each group of GA, the thickness and width are the same; the emissivity, specific heat and real thermal diffusivity can be taken equal. From equation (2), the radiation effect in the measured thermal diffusivity is proportional to $L^{2}$ (L: sample length). By plotting the measured thermal diffusivity $\left(\alpha_{m}\right)$ against $L^{2}$ at each temperature and linear fitting to $L^{2}=0$, we are able to subtract the radiation effect and identify the real thermal diffusivity $\left(\alpha_{\text {real }}\right)$. This method has been demonstrated in our previous work. ${ }^{32}$ The same method is employed to subtract the radiation effect to obtain the real thermal conductivity $(\kappa)$ of GAs. The inset in figure 2 (d) shows one of the linear fitting process for obtaining the real thermal conductivity of GA (group 1 at real temperature of $298.6 \mathrm{~K}$ ).

\section{Extremely low thermal conductivity}

The real thermal conductivity of GAs is plotted out in Figure 3 (a). As is seen in the figure, $\kappa$ for the two groups are extremely low. At RT, $\kappa$ is $4.7 \times 10^{-3}$ and $5.9 \times 10^{-3} \mathrm{~W} \cdot \mathrm{m}^{-1} \cdot \mathrm{K}^{-1}$ for group 2 and group 1 respectively, which is similar to the reported lowest value for silica aerogel at moderate vacuum $\left(0.004 \mathrm{~W} \cdot \mathrm{m}^{-1} \cdot \mathrm{K}^{-1}\right)$. This value is much lower than the disordered, layered $\mathrm{WSe}_{2}$ crystals $^{33}\left(0.05 \mathrm{~W} \cdot \mathrm{m}^{-1} \cdot \mathrm{K}^{-1}\right.$ at $\left.\mathrm{RT}\right)$; and microcrystalline [6,6]-phenyl C61-butyric acid methyl 
ester $(\mathrm{PCBM})$ thin films $\left(0.03 \pm 0.003 \mathrm{~W} \cdot \mathrm{m}^{-1} \cdot \mathrm{K}^{-1}\right.$ at $\left.\mathrm{RT}\right){ }^{34}$ They were reported as the lowest thermal conductivity materials for a full dense solid and has been used as a new insulating material in recent years. As temperature goes down, $\kappa$ quickly decreases and is lower than $10^{-3}$ $\mathrm{W} \cdot \mathrm{m}^{-1} \cdot \mathrm{K}^{-1}$ at temperatures below $86 \mathrm{~K}$. At temperature of $46 \mathrm{~K}$, the thermal conductivity of the two groups even decreases to $7.15 \times 10^{-4}$ and $2.20 \times 10^{-4} \mathrm{~W} \cdot \mathrm{m}^{-1} \cdot \mathrm{K}^{-1}$ respectively. The reason that the thermal insulation performance in our report is better compared to Wicklein, et. al's anisotropic foams ${ }^{18}$ is due to the air conduction effect. In our work, the thermal characterization is conducted in vacuum environment (air pressure less than 5 mTorr). Using Maxwell's model ${ }^{35}$ for effective thermal conductivity of a mixture, the thermal conductivity of our GA with air conduction effect is estimated around $25.85 \mathrm{~mW} \cdot \mathrm{m}^{-1} \cdot \mathrm{K}^{-1}$. Due to the scattering effect of the cell walls, the mean free path of air within pores could be much smaller than that in free space $(\sim 200$ $\mathrm{nm})$. Thus, the real thermal conductivity should be even lower since the thermal conductivity of air within the pores of GA can be reduced dramatically compared to that in free space. Compared to Fan, et al.' work, ${ }^{17}$ their GA material has a much higher density $\left(16-41 \mathrm{mg} \cdot \mathrm{cm}^{-3}\right)$ than our GA $\left(4 \mathrm{mg} \cdot \mathrm{cm}^{-3}\right)$. This will make their sample have a higher thermal conductivity than ours.

The trends of $\kappa$ are very similar for the two groups. From the $\kappa-T$ evolution, $\kappa$ of group 1 decreases from $5.9 \times 10^{-3} \mathrm{~W} \cdot \mathrm{m}^{-1} \cdot \mathrm{K}^{-1}$ at $299 \mathrm{~K}$ to $4.3 \times 10^{-4} \mathrm{~W} \cdot \mathrm{m}^{-1} \cdot \mathrm{K}^{-1}$ at $36 \mathrm{~K} ; \kappa$ of group 2 decreases from $4.7 \times 10^{-3} \mathrm{~W} \cdot \mathrm{m}^{-1} \cdot \mathrm{K}^{-1}$ at $299 \mathrm{~K}$ to $2.2 \times 10^{-4} \mathrm{~W} \cdot \mathrm{m}^{-1} \cdot \mathrm{K}^{-1}$ at $46 \mathrm{~K}$. This is an interesting phenomenon since it is completely contrary to the thermal conductivity of graphite ${ }^{36}$ and our previously reported graphene foam $(\mathrm{GF}){ }^{37}$ The inset in Figure 3 (a) shows the thermal conductivity of pyrolytic graphite ${ }^{36}$ for comparison. As seen in the inset, $\kappa$ of graphite generally increases from RT to the peak temperature (normally $100 \mathrm{~K}$ ), and then decreases after the peak. 
The peak position is mainly determined by the defect level in graphite samples. As the perfection and order of structure improve, the peak shifts to a lower temperature. ${ }^{38}$ For our GAs samples, the thermal conductivity for the two groups decreases all the way down to $40 \mathrm{~K}$ with some data fluctuation at very low temperatures. This indicates the highly disordered structure in the GA samples. The data at temperatures lower than $40 \mathrm{~K}$ should be used with less confidence due to the large data fluctuations. $\kappa$ of group 2 is a little smaller than that of group 1 , which is reasonable considering the lower density of group $2\left(4.2 \mathrm{mg} \cdot \mathrm{cm}^{-3}\right.$ and $3.9 \mathrm{mg} \cdot \mathrm{cm}^{-3}$ for group 1 and group 2 respectively).

Based on the model of Schuetz et al, ${ }^{39}$ a correlation for the thermal conductivity of porous media has been demonstrated reliable as $\kappa_{G}=3 \kappa_{G A} / \varphi$. Using this equation, the intrinsic thermal conductivity of GAs without the porosity effect $\left(\kappa_{G}\right)$ can be estimated. In this equation, $\kappa_{G A}$ is the thermal conductivity of porous graphene aerogels, and $\varphi=\rho_{G A} / \rho_{G}$ is the volume fraction of the solid phase in the GAs sample. For group 1 and group 2, the density is measured as $4.2 \mathrm{mg} \cdot \mathrm{cm}^{-3}$ and $3.9 \mathrm{mg} \cdot \mathrm{cm}^{-3}$ respectively. Using density of graphite $\rho=2200 \mathrm{mg} \cdot \mathrm{cm}^{-3}, \varphi$ of two groups of GAs are estimated as 0.0019 and 0.0018 . The porosity of the two samples is accordingly $99.81 \%$ and $99.82 \%$. The result of $\kappa_{G}$ is plotted in figure $3(\mathrm{~b})$. From our calculation, $\kappa_{G}$ is $9.3 \mathrm{~W} \cdot \mathrm{m}^{-1} \cdot \mathrm{K}^{-1}$ at RT and decreases to $1.4 \mathrm{~W} \cdot \mathrm{m}^{-1} \cdot \mathrm{K}^{-1}$ at $10.4 \mathrm{~K}$ for group $1 ; \kappa_{G}$ is lower than $8.0 \mathrm{~W} \cdot \mathrm{m}^{-1} \cdot \mathrm{K}^{-1}$ for group 2 at temperature from $46 \mathrm{~K}$ to $299 \mathrm{~K}$. This calculation proves the very low intrinsic thermal conductivity of the r-GO framework. In addition to the contribution from the high porosity, the low thermal conductivity of the r-GO framework is also responsible for the ultralow thermal conductivity of GAs. The intrinsic thermal conductivity of the solid phase inside the GAs is two orders of magnitude lower than the ultra-high thermal conductivity of graphene 

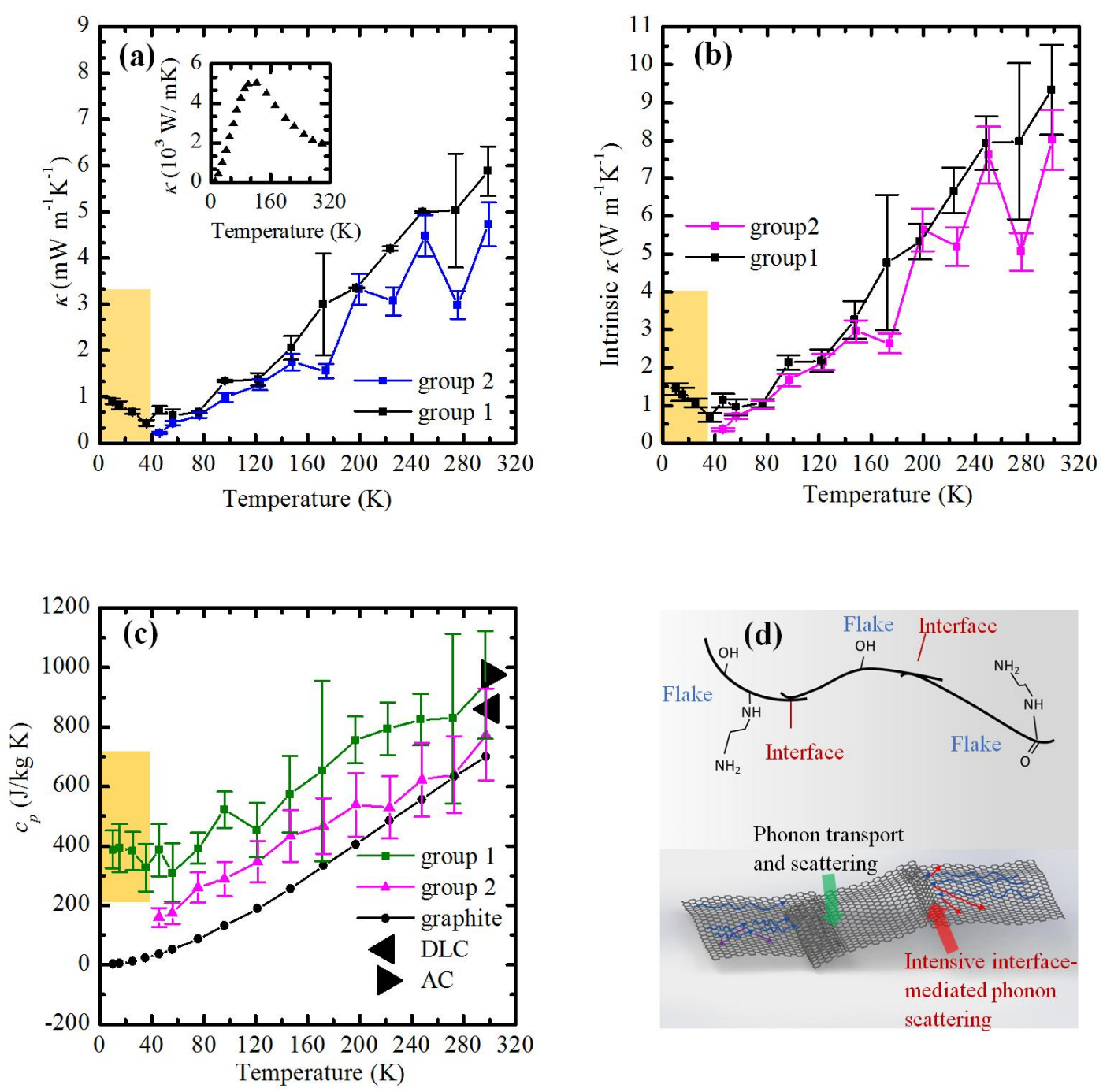

Figure 3. (a) The thermal conductivity of the two groups of GAs. The inset shows the $\kappa$ of pyrolytic graphite for comparison. (b) The intrinsic thermal conductivity of GAs by taking out the porosity effect. (c) The specific heat of the two GA samples. The literature data for graphite and amorphous carbon are also plotted for comparison. The data inside the yellow rectangular is less reliable due to the large data fluctuation at very low temperatures. (d) The schematic drawing that illustrates the heat transfer process inside GA. At the interfaces of the flakes, intensive interface-mediated phonon scatterings occur. 
$\left(\sim 5000 \mathrm{~W} \cdot \mathrm{m}^{-1} \cdot \mathrm{K}^{-1}\right)$. It has been reported that substitution of just $1 \%$ of carbon atoms with nitrogen caused $59.2 \%$ reduction in thermal conductivity at $300 \mathrm{~K}$. The $\mathrm{N}$ dopants significantly increase the phonon scattering in GA and contribute to the ultralow thermal conductivity. However, large residual nitrogen content inside the GA would sacrifice the mechanical strength of GA.

The specific heat $\left(c_{p}\right)$ against temperature profile provides more hints about the structure of the GA. $c_{p}$ at different temperatures is obtained using the measured thermal diffusivity $\alpha_{m}$ and measured thermal conductivity $\kappa_{m}$ as $c_{p}=\kappa_{m} / \rho \alpha_{m}$, in which $\rho$ is the density of the GA sample. Figure 3 (c) shows the average specific heat from two groups of GAs compared with that of high-purity Acheson graphite, ${ }^{40}$ diamond-like carbon films (DLC) and amorphous carbon (AC). ${ }^{41}$ As temperature goes down from RT to $45 \mathrm{~K}, c_{p}$ of GA decrease linearly in both cases. The trends and slopes are both very similar to that of graphite. As temperature goes to zero, the specific heat should go to zero. The pattern at very low temperature is similar to that of organic materials. ${ }^{42}$ The data below $45 \mathrm{~K}$ goes up a little, which is due to the error resulting from large data fluctuation at very low temperatures [see figure 2 (b)]. The $c_{p}$ of GAs is a little higher than that of graphite. The difference between the value of GAs and graphite are largely attributed to two factors: the error in the GAs' density measurement and the difference between the structure of r-GO and that of highly oriented graphite. The unavoidable error when measuring the size of the GA films could result in errors of the density, which makes the specific heat value overestimated. Besides, the XPS result indicates there are many extra atoms including oxygen and nitrogen and functional groups at the surfaces of GAs, which distort the atomic positions and increase the structure disorder. Thus, the structure of the GAs is different from that of graphite. 
There has not been any experimental measurements about the specific heat of reduced graphite oxide to our best knowledge. In literatures, $c_{p}$ of GO has always been assumed similar to that of graphite ${ }^{43}$ or amorphous carbon. ${ }^{44}$ Our $c_{p}$ for GAs is very close to the value for DLC and AC. From the XRD spectra, the peaks of GAs are wide and not obvious. This suggests that the GAs are not well-crystallized graphitic material and contains large quantity of amorphous structure. It has been reported that the specific heat of amorphous materials exceeds that of the crystalline form. ${ }^{45,46}$ The much more amorphous structure of GAs than the highly ordered Acheson graphite could also contribute to the higher heat capacity of GAs. The different microwave heating time (2 minutes for group 1 and 4 minutes for group 2) leaves the two groups of samples with different amount of nitrogen-containing groups. ${ }^{22}$ Microwave heating removed more functional groups for group 2, which results in a larger $c_{p}$ of group 1 than that of group 2.

\section{The underlying mechanism and temperature-dependent phonon scattering}

Figure 3 (d) presents the schematic drawing of the heat transfer mechanism inside the GA. Thermal transport inside GA is controlled by phonons transport among r-GO sheets. During the transport, phonons are not only scattered within single flake by phonons, defects and grain boundaries, but also scattered at the interfaces of neighboring r-GO flakes. The r-GO sheets are self-assembled driven by the increasing hydrophobicity and the $\pi-\pi$ interaction among r-GO sheets during the chemical reduction. The interface between the r-GO sheets is through $\pi-\pi$ interaction with small bonding areas. The scattering intensity at interfaces can be very high.

To better understand the underlying mechanism for the ultralow thermal conductivity, the thermal diffusivity of GAs at different temperatures is measured and analyzed. Figure 4 (a) 
shows the real thermal diffusivity of the two groups of GAs samples. From RT to low temperatures, both $\alpha$ change with temperature very slowly in a small scale. $\alpha$ of group 1 decreases from $8.46 \times 10^{-7} \mathrm{~m}^{2} / \mathrm{s}$ at $297 \mathrm{~K}$ to $3.0 \times 10^{-7} \mathrm{~m}^{2} / \mathrm{s}$ at $10 \mathrm{~K} ; \alpha$ of group 2 ranges from $1.62 \times 10^{-6} \mathrm{~m}^{2} / \mathrm{s}$ at $297 \mathrm{~K}$ to $8.3 \times 10^{-7} \mathrm{~m}^{2} / \mathrm{s}$ at $45 \mathrm{~K}$. The decrease of $\alpha$ is relatively trivial compared to the previously reported thermal diffusivity change of graphene foam $(\mathrm{GF})^{37}$ and pyrolytic graphite $^{36}$ [Figure 4 (a)]. As shown at the bottom panel of figure 4 (a), $\alpha$ of GF and graphite follows a quick increasing behavior as temperature goes down, and finally becomes stable. In contrast, both $\alpha$ of our GAs stay almost constant with a small decrease in the low temperature range. This result uncovers a completely different dominant thermal transport mechanism, distinguishing our GAs from other graphene-based materials.

We speculate that the main effect controlling the thermal transport is the thermal contact resistance, rather than the phonon-phonon scattering. From single relaxation time approximation, a classical model for phonon thermal conductivity can be expressed as: $\kappa=1 / 3 \rho c_{p} v^{2} \tau$. Here, $v$ is the effective and averaged phonon velocity. $\tau$ is an averaged relaxation time for phonon scatterings, inversely proportional to phonon scattering intensity. This equation can be expressed in terms of thermal reffusivity (inverse of thermal diffusivity) as: $\alpha^{-1}=3 / v^{2} \tau=\Theta_{0}+3 / v^{2} \tau_{u}$, in which $\Theta_{0}$ is the residual thermal reffusivity (induced by defects), and $\tau_{u}$ is the relaxation time from Umklapp scattering. As temperature goes down, the phonon population participating in the Umklapp scattering drops with the decreasing temperature, resulting in an increased $\tau_{u}$ correspondingly. As temperature approaches absolute zero, the Umklapp scattering term vanishes, and $\alpha^{-1}$ reaches a constant value $\Theta_{0}$, which is controlled by the residual defect and boundary scattering. For GF and graphite, the thermal contact resistance at interfaces is relatively 

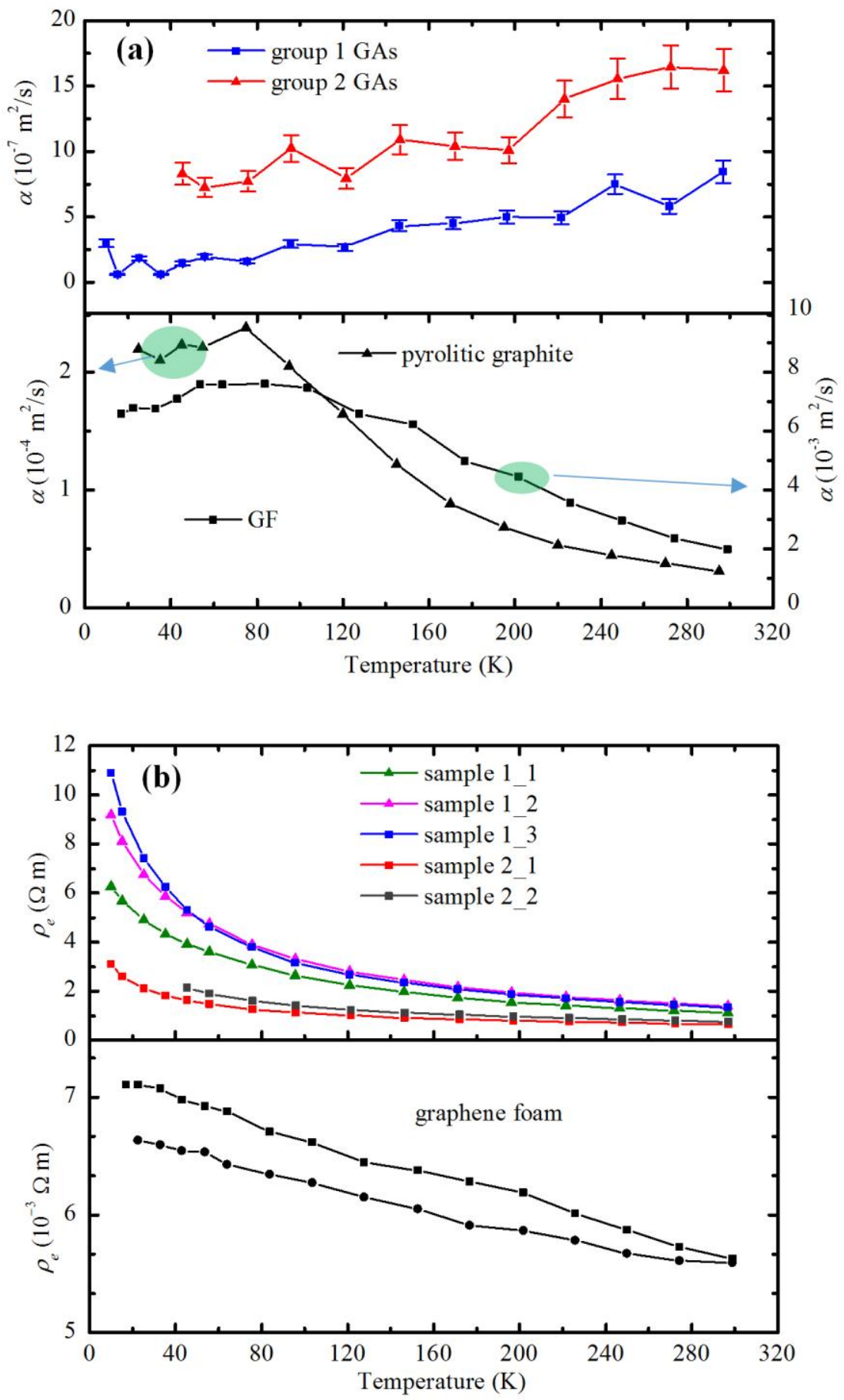

Figure 4. (a) The thermal diffusivity of the two groups of GAs compared with that of graphene foam (GF) and pyrolytic graphite. (b) The electrical resistivity against temperature for the five GA samples. The linear $\rho_{e}-T$ data of graphene foam is also plotted at the bottom panel for comparison. 
small due to their continuous and covalently bonded structure. Umklapp scattering of phonons mainly controls the thermal transport. Thus, their $\alpha$ first increases and then becomes stable when temperature goes down to the $0 \mathrm{~K}$ limit. This phenomenon was also observed in other bulk materials, such as DNA, silicon, germanium, $\mathrm{NaCl}$ and $\mathrm{NaF}^{47}$ In contrast, for GAs, the r-GO sheets are self-assembled during the chemical reduction. The contacting areas of one flake with both the medium and neighboring flakes are small. In addition, the connecting mechanism among the neighboring self-assembled $\mathrm{r}-\mathrm{GO}$ sheets is mainly $\pi-\pi$ interaction, which is a weak electrostatic interaction between aromatic rings. Therefore, the thermal contact resistance at the interfaces between flakes is much larger.

A model for heat transfer in GA can be expressed as: $l_{f} / \kappa_{e f f}=l_{f} / \kappa_{c}+R$, where $l_{f}$ is the average flake size, $\kappa_{\text {eff }}$ is the effective thermal conductivity of GA, and $\kappa_{c}$ is the thermal conductivity within r-Go flake, which includes the grain boundary thermal resistance. $R$ is the interfacial thermal contact resistance between neighboring flakes. Multiplying the specific heat $\rho c_{p}$ of GAs at both sides, we can express the equation in terms of thermal diffusivity as $\alpha_{\text {eff }}^{-1}=R \rho c_{p} / l_{f}+\alpha_{c}^{-1}$, where $\alpha_{\text {eff }}$ is the effective thermal diffusivity and $\alpha_{c}$ is the intrinsic thermal diffusivity of r-GO flake. Combining the above thermal reffusivity equation, an expression can be deduced for the thermal contact conductance at interfaces: $R=A / \rho c_{p} v$, where $A$ is a constant. When the thermal contact resistance at interface is very large: $\mathrm{R} \gg l_{f} / \kappa_{c}$, the equation can be simplified as $\alpha_{\text {eff }}=A v l_{f}$, in which the phonon velocity $v$ and $l_{f}$ are weakly temperature dependent. Thus, the thermal diffusivity of GAs stays almost unchanged. The dominating mechanism controlling thermal transport in GAs is the interfacial thermal contact resistance among the flakes. 
In spite of the above analysis, there is still a small decrease in the thermal diffusivity of GA with decreased temperature. We ascribe this decrease to the thermal expansion and thermal radiation among the r-GO sheets inside GAs. As shown at the bottom panel of figure 4 (b), $\rho_{e}$ of GF is inversely proportional to the temperature as expected, ${ }^{37}$ which is the common behavior of graphene based material. $\rho_{e}$ of GAs is very much different from the electrical resistivity of GF. As plotted in Figure 4 (b), $\rho_{e}$ presents a nonlinear increasing behavior and increases exponentially with reduced temperature at low temperatures. The fast increasing electrical resistivity of GAs at low temperatures indicates the worsened contact among the r-GO sheets due to the temperature decrease. The aggravated contact inevitably increases the thermal and electrical contact resistance. The r-GO sheets are self-assembled during the chemical reduction. The connection among sheets is randomly oriented and stress-balanced. While as temperature goes down, the thermal expansion of the r-GO sheets results in thermal strains inside the samples. The expansion deteriorates the contact among r-GO sheets and contributes to the decreasing thermal diffusivity consequently. In addition, the radiation effect inside the pores could also contribute to the decreasing thermal diffusivity of GAs. Pores from tens to hundreds of micrometers are formed within the r-GO framework. Within these pores, thermal radiation occurs among the neighboring $\mathrm{r}-\mathrm{GO}$ sheets. The radiation irradiance follows a behavior of $j^{*}=\varepsilon \sigma T^{4}$. As temperature goes down, the radiation energy flux decreases by $\sim T^{4}$, so the thermal conductivity contribution from radiation decreases by $\sim T^{3}$, which is faster than the specific heat of GAs (linear relation with $T$ ). This results in that part of effective thermal diffusivity decreases by $\sim T^{2}$. The evident separation of the two groups' data further proves the structure difference for the two groups of GAs. $\rho_{e}$ for samples of group 1 is obviously larger than that of group 2, further indicating the more defected structure of group 1 GAs. Besides, the 
variation of $\rho_{e}$ for group 2 at low temperatures is relatively small, which proposes that the contact deterioration has smaller effect to group 2 than group 1. This further demonstrates the different defect levels between the two groups of samples, which we also observe in the thermal conductivity profile.

\section{Conclusion}

In summary, we synthesized graphene aerogels material with an extremely low thermal conductivity using an improved chemical reduction method. The resulting GAs has a very low density (2-6 $\mathrm{mg} \mathrm{cm}^{3}$ ) and good elasticity. By employing the SET technique, we measured the thermal conductivity from RT to as low as $10.4 \mathrm{~K}$ for the two groups of GAs (density of 4.2 and $3.9 \mathrm{mg} \mathrm{cm}^{3}$ respectively). The thermal conductivity of our GA is extremely low (down to $2 \times 10^{-4}$ $-4 \times 10^{-4} \mathrm{~W} \cdot \mathrm{m}^{-1} \cdot \mathrm{K}^{-1}$ at low temperatures and $4.7 \times 10^{-3}-5.9 \times 10^{-3} \mathrm{~W} \cdot \mathrm{m}^{-1} \cdot \mathrm{K}^{-1}$ at $\mathrm{RT}$ ), which makes it a very promising material for thermal insulation. The thermal diffusivity is further characterized using the TET technique. The thermal diffusivity stays almost constant with a little decrease with the decreased temperature, revealing the dominating effect of thermal contact resistance for sustaining the thermal transport in GAs. The exponentially increasing electrical resistivity (against decreased temperature) indicates the contact among r-GO sheets is worsened as temperature goes down. The specific heat calculated from the experimental data shows a very similar pattern as that of graphite. The value is close to that of amorphous carbon. The results strongly demonstrate the amorphous structure within the GAs, which is also revealed by XRD characterization. The extremely low thermal conductivity uncovered in this work is for GAs of a density around $4 \mathrm{mg} \mathrm{cm}^{-3}$. We predict when the density of GAs is reduced to the level of $0.16 \mathrm{mg}$ $\mathrm{cm}^{-3}$ (lightest reported density for GAs to date), the thermal conductivity of GAs could be 
significantly reduced down to the order of $10^{-4} \mathrm{~W} \cdot \mathrm{m}^{-1} \cdot \mathrm{K}^{-1}{ }^{1}$ at $\mathrm{RT}$, and $10^{-6} \sim 10^{-5} \mathrm{~W} \cdot \mathrm{m}^{-1} \cdot \mathrm{K}^{-1}$ at temperatures around $10 \mathrm{~K}$. This will make the graphene aerogel an unprecedented insulating material for thermal protection, especially under vacuum conditions (e.g. astronautics areas).

\section{Acknowledgements}

Support of this work by Army Research Office (W911NF-12-1-0272), Office of Naval Research (N000141210603), and National Science Foundation (CBET1235852, CMMI1264399, and CMMI1200397) is gratefully acknowledged. X.W. thanks the partial support of the "Eastern Scholar" Program of Shanghai, China. Y.X is supported by the China Scholarship Council. 


\section{References}

1. Worsley, M. A.; Pauzauskie, P. J.; Olson, T. Y.; Biener, J.; Satcher, J. H.; Baumann, T. F. Synthesis of Graphene Aerogel with High Electrical Conductivity. J Am Chem Soc 2010, 132 (40), 14067-14069.

2. Zhang, X. T.; Sui, Z. Y.; Xu, B.; Yue, S. F.; Luo, Y. J.; Zhan, W. C.; et al. Mechanically strong and highly conductive graphene aerogel and its use as electrodes for electrochemical power sources. J Mater Chem 2011, 21 (18), 6494-6497.

3. Sui, Z. Y.; Meng, Q. H.; Zhang, X. T.; Ma, R.; Cao, B. Green synthesis of carbon nanotubegraphene hybrid aerogels and their use as versatile agents for water purification. J Mater Chem 2012, 22 (18), 8767-8771.

4. Li, J.; Wang, F.; Liu, C. Y. Tri-isocyanate reinforced graphene aerogel and its use for crude oil adsorption. J Colloid Interf Sci 2012, 382 (1), 13-16.

5. Wu, Z. S.; Sun, Y.; Tan, Y. Z.; Yang, S. B.; Feng, X. L.; Mullen, K. Three-Dimensional Graphene-Based Macro- and Mesoporous Frameworks for High-Performance Electrochemical Capacitive Energy Storage. J Am Chem Soc 2012, 134 (48), 19532-19535.

6. Li, J. H.; Li, J. Y.; Meng, H.; Xie, S. Y.; Zhang, B. W.; Li, L. F.; Ma, H. J.; Zhang, J. Y.; Yu, M. Ultra-light, compressible and fire-resistant graphene aerogel as a highly efficient and recyclable absorbent for organic liquids. J Mater Chem A 2014, 2 (9), 2934-2941.

7. Xu, X.; Li, H.; Zhang, Q. Q.; Hu, H.; Zhao, Z. B.; Li, J. H.; Li, J. Y.; Qiao, Y.; Gogotsi, Y. Self-Sensing, Ultra light, and Conductive 3D Graphene/Iron Oxide Aerogel Elastomer Deformable in a Magnetic Field. Acs Nano 2015, 9 (4), 3969-3977.

8. Zhang, Q. Q.; Xu, X.; Li, H.; Xiong, G. P.; Hu, H.; Fisher, T. S. Mechanically robust honeycomb graphene aerogel multifunctional polymer composites. Carbon 2015, 93, 659670.

9. Dorcheh, A. S.; Abbasi, M. H. Silica aerogel; synthesis, properties and characterization. $J$ Mater Process Tech 2008, 199 (1), 10-26.

10. Baetens, R.; Jelle, B. P.; Gustavsen, A. Aerogel insulation for building applications: A stateof-the-art review. Energ Buildings 2011, 43 (4), 761-769.

11. Wei, T. Y.; Chang, T. F.; Lu, S. Y.; Chang, Y. C. Preparation of monolithic silica aerogel of low thermal conductivity by ambient pressure drying. J Am Ceram Soc 2007, 90 (7), 20032007.

12. Deng, Z. S.; Wang, J.; Wu, A. M.; Shen, J.; Zhou, B. High strength $\mathrm{SiO}_{2}$ aerogel insulation. J Non-Cryst Solids 1998, 225, 101-104.

13. Zeng, S. Q.; Hunt, A.; Greif, R. Geometric structure and thermal conductivity of porous medium silica aerogel. J Heat Trans-T Asme 1995, 117 (4), 1055-1058.

14. Poco, J. F.; Satcher, J. H.; Hrubesh, L. W. Synthesis of high porosity, monolithic alumina aerogels. J Non-Cryst Solids 2001, 285 (1), 57-63.

15. Fan, Z.; Marconnet, A.; Nguyen, S. T.; Lim, C. Y. H.; Duong, H. M. Effects of heat treatment on the thermal properties of highly nanoporous graphene aerogels using the infrared microscopy technique. Int J Heat Mass Tran 2014, 76, 122-127.

16. Balandin, A. A.; Ghosh, S.; Bao, W. Z.; Calizo, I.; Teweldebrhan, D.; Miao, F., et al. Superior thermal conductivity of single-layer graphene. Nano Lett 2008, 8 (3), 902-907.

17. Fan, Z.; Tng, D. Z. Y.; Lim, C. X. T.; Liu, P.; Nguyen, S. T.; Xiao, P. F.; Marconnet, A.; Lim, C. Y. H.; Duong, H. M. Thermal and electrical properties of graphene/carbon nanotube aerogels. Colloid Surface A 2014, 445, 48-53. 
18. Wicklein, B.; Kocjan, A.; Salazar-Alvarez, G.; Carosio, F.; Camino, G.; Antonietti, M.; Bergstrom, L. Thermally insulating and fire-retardant lightweight anisotropic foams based on nanocellulose and graphene oxide. Nat Nanotechnol 2015, 10 (3), 277-283.

19. Tang, Z. H.; Shen, S. L.; Zhuang, J.; Wang, X. Noble-Metal-Promoted Three-Dimensional Macroassembly of Single-Layered Graphene Oxide. Angew Chem 2010, 122 (27), 4707-4711.

20. Wu, Z. S.; Yang, S. B.; Sun, Y.; Parvez, K.; Feng, X. L.; Mullen, K. 3D Nitrogen-Doped Graphene Aerogel-Supported $\mathrm{Fe}_{3} \mathrm{O}_{4}$ Nanoparticles as Efficient Eletrocatalysts for the Oxygen Reduction Reaction. J Am Chem Soc 2012, 134 (22), 9082-9085.

21. Sun, H. Y.; Xu, Z.; Gao, C. Multifunctional, Ultra-Flyweight, Synergistically Assembled Carbon Aerogels. Adv Mater 2013, 25 (18), 2554-2560.

22. Hu, H.; Zhao, Z. B.; Wan, W. B.; Gogotsi, Y.; Qiu, J. S. Ultralight and Highly Compressible Graphene Aerogels. Adv Mater 2013, 25 (15), 2219-2223.

23. Some, S.; Kim, Y.; Yoon, Y.; Yoo, H.; Lee, S.; Park, Y., et al. High-Quality Reduced Graphene Oxide by a Dual-Function Chemical Reduction and Healing Process. Sci Rep-Uk 2013, 3.

24. Stankovich, S.; Dikin, D. A.; Piner, R. D.; Kohlhaas, K. A.; Kleinhammes, A.; Jia, Y., et al. Synthesis of graphene-based nanosheets via chemical reduction of exfoliated graphite oxide. Carbon 2007, 45 (7), 1558-1565.

25. Zhang, J. L.; Yang, H. J.; Shen, G. X.; Cheng, P.; Zhang, J. Y.; Guo, S. W. Reduction of graphene oxide via L-ascorbic acid. Chem Commun 2010, 46 (7), 1112-1114.

26. Tuinstra, F.; Koenig, J. L. Raman Spectrum of Graphite. J Chem Phys 1970, 53 (3), 11261130.

27. Park, S.; An, J.; Potts, J. R.; Velamakanni, A.; Murali, S.; Ruoff, R. S. Hydrazine-reduction of graphite- and graphene oxide. Carbon 2011, 49 (9), 3019-3023.

28. Cui, P.; Lee, J.; Hwang, E.; Lee, H. One-pot reduction of graphene oxide at subzero temperatures. Chem Commun 2011, 47 (45), 12370-12372.

29. Huh, S. H. Thermal Reduction of Graphene Oxide. Physics and Applications of Graphene Experiments 2011, 73-90.

30. Mahmood, N.; Zhang, C. Z.; Yin, H.; Hou, Y. L. Graphene-based nanocomposites for energy storage and conversion in lithium batteries, supercapacitors and fuel cells. J Mater Chem A 2014, 2 (1), 15-32.

31. Guo, J. Q.; Wang, X. W.; Wang, T. Thermal characterization of microscale conductive and nonconductive wires using transient electrothermal technique. Journal of Applied Physics 2007, 101 (6), 063537.

32. Lin, H.; Xu, S.; Wang, X. W.; Mei, N. Significantly reduced thermal diffusivity of freestanding two-layer graphene in graphene foam. Nanotechnology 2013, 24 (41), 415706.

33. Chiritescu, C.; Cahill, D. G.; Nguyen, N.; Johnson, D.; Bodapati, A.; Keblinski, P., et al. Ultralow thermal conductivity in disordered, layered $\mathrm{WSe}_{2}$ crystals. Science 2007, 315 (5810), 351-353.

34. Duda, J. C.; Hopkins, P. E.; Shen, Y.; Gupta, M. C. Exceptionally Low Thermal Conductivities of Films of the Fullerene Derivative PCBM. Phys Rev Lett 2013, 110 (1), 015902.

35. Wang, X. W.; Xu, X. F.; Choi, S. U. S. Thermal conductivity of nanoparticle-fluid mixture. J Thermophys Heat Tr 1999, 13 (4), 474-480

36. Ho, C. Y.; Powell, R. W.; Liley, P. E. Thermal conductivity of the elements: a comprehensive review. American Chemical Society: Washington, 1975; p 796. 
37. Y.S. Xie; Z.L. Xu; S. Xu; Z. Cheng; N. Hashemi; C. Deng, et al. The defect level and ideal thermal conductivity of graphene uncovered by residual thermal reffusivity at the $0 \mathrm{~K}$ limit. Nanoscale 2015, 7 (22), 10101-10110.

38. Klett, J. W.; McMillan, A. D.; Gallego, N. C.; Walls, C. A. The role of structure on the thermal properties of graphitic foams. J Mater Sci 2004, 39 (11), 3659-3676.

39. Schuetz, M. A.; Glicksman, L. R. A Basic Study of Heat-Transfer through Foam Insulation. $J$ Cell Plast 1984, 20 (2), 114-121.

40. Desorbo, W.; Tyler, W. W. The Specific Heat of Graphite from 13-Degrees-K to 300Degrees-K. J Chem Phys 1953, 21, 1660-1663.

41. Hakovirta, M.; Vuorinen, J. E.; He, X. M.; Nastasi, M.; Schwarz, R. B. Heat capacity of hydrogenated diamond-like carbon films. Appl Phys Lett 2000, 77 (15), 2340-2342.

42. Chang, S. S. Heat-Capacities of Polyethylene from 2 to $360 \mathrm{~K}$.П. Two High-Density Linear Polyethylene Samples and Thermodynamic Properties of Crystalline Linear Polyethylene. $J$ Res Nbs a Phys Ch 1974, 3, 387-400.

43. McAllister, M. J.; Li, J. L.; Adamson, D. H.; Schniepp, H. C.; Abdala, A. A.; Liu, J., et al. Single sheet functionalized graphene by oxidation and thermal expansion of graphite. Chem Mater 2007, 19 (18), 4396-4404.

44. Zhou, Y.; Bao, Q. L.; Varghese, B.; Tang, L. A. L.; Tan, C. K.; Sow, C. H., et al. Microstructuring of Graphene Oxide Nanosheets Using Direct Laser Writing. Adv Mater 2010, 22 (1), 67-71.

45. Johnston, H. L.; Hersh, H. N.; Kerr, E. C. Low Temperature Heat Capacities of Inorganic Solids. $1 \mathrm{~V}$. The Heat Capacity of Pure Elementary Boron in Both Amorphous and Crystalline Conditions between 13 and $305^{\circ} \mathrm{K}$. Some Free Energies of Formation. $J$ Am Chem Soc 1951, 73 (3), 1112-1117.

46. Fulde, P.; Wagner, H. Low-Temperature Specific Heat and Thermal Conductivity of Noncrystalline Solids. Phys Rev Lett 1971, 27 (19), 1280.

47. Xu, Z. L.; Wang, X. W.; Xie, H. Q. Promoted electron transport and sustained phonon transport by DNA down to 10 K. Polymer 2014, 55 (24), 6373-6380. 